\title{
Possible explanations of the Maunder minimum from a flux transport dynamo model
}

\author{
Bidya Binay Karak and Arnab Rai Choudhuri \\ Department of Physics, Indian Institute of Science, Bangalore-560012 \\ email: bidya_karak@physics.iisc.ernet.in, \\ arnab@physics.iisc.ernet.in
}

\begin{abstract}
We propose that at the beginning of the Maunder minimum the poloidal field or amplitude of meridional circulation or both fell abruptly to low values. With this proposition, a flux transport dynamo model is able to reproduce various important aspects of the historical records of the Maunder minimum remarkably well.
\end{abstract}

Keywords. Sun: activity, sun: magnetic field, meridional circulation

\section{Introduction}

One important aspect of the solar cycle is the Maunder minimum during 1645-1715 when the solar activity was strongly reduced (Ribes \& Nesme-Ribes 1993). It was not an artifact of few observations, but a real phenomenon (Hoyt \& Schatten 1996). From the study of historical data (Ribes \& Nesme-Ribes 1993), it has been confirmed that the sunspot numbers in both the hemisphere fell abruptly to nearly zero value at the beginning of the Maunder minimum, whereas a few sunspots appeared in the southern hemisphere during the last phase. It is also established from the cosmogenic isotopes data (Beer et al. 1998; Miyahara et al. 2004) that the cyclic oscillations of solar activity continued in the heliosphere at a weaker level during the Maunder minimum, but with a period of 13-15 years instead of the regular 11-year period.

The most promising model of studying solar cycle at present is the flux transport dynamo model (Choudhuri et al. 1995; Durney 1995; Dikpati \& Charbonneau 1999; Chatterjee et al. 2004). The main sources of irregularities in this model are the stochastic fluctuations in the Babcock-Leighton process of poloidal field generation (Choudhuri 1992; Choudhuri et al. 2007) and the stochastic fluctuations of meridional circulation (hereafter MC) (Hathaway 1996). Therefore we propose that the polar field or amplitude of $\mathrm{MC}$ or both decreased at the beginning of Maunder minimum. With this proposition, we use a flux transport dynamo model to reproduce a Maunder minimum. The details of this work can be found in Choudhuri \& Karak (2009) and Karak (2010).

\section{Methodology}

We cary out all the analyses with the flux transport dynamo model described in Chatterjee et al. (2004). To reproduce the Maunder minimum, we perform the following three separate sets of experiments. Similar to Choudhuri et al. (2007), first, we decrease the polar field above $0.8 R_{\odot}$ by a factor $\gamma$ after stopping the code at a solar minimum. We change the polar field by different amount in two hemispheres. In northern hemisphere, we take $\gamma=0.0$, whereas in southern hemisphere, it is 0.4 . In addition, in this calculation, 

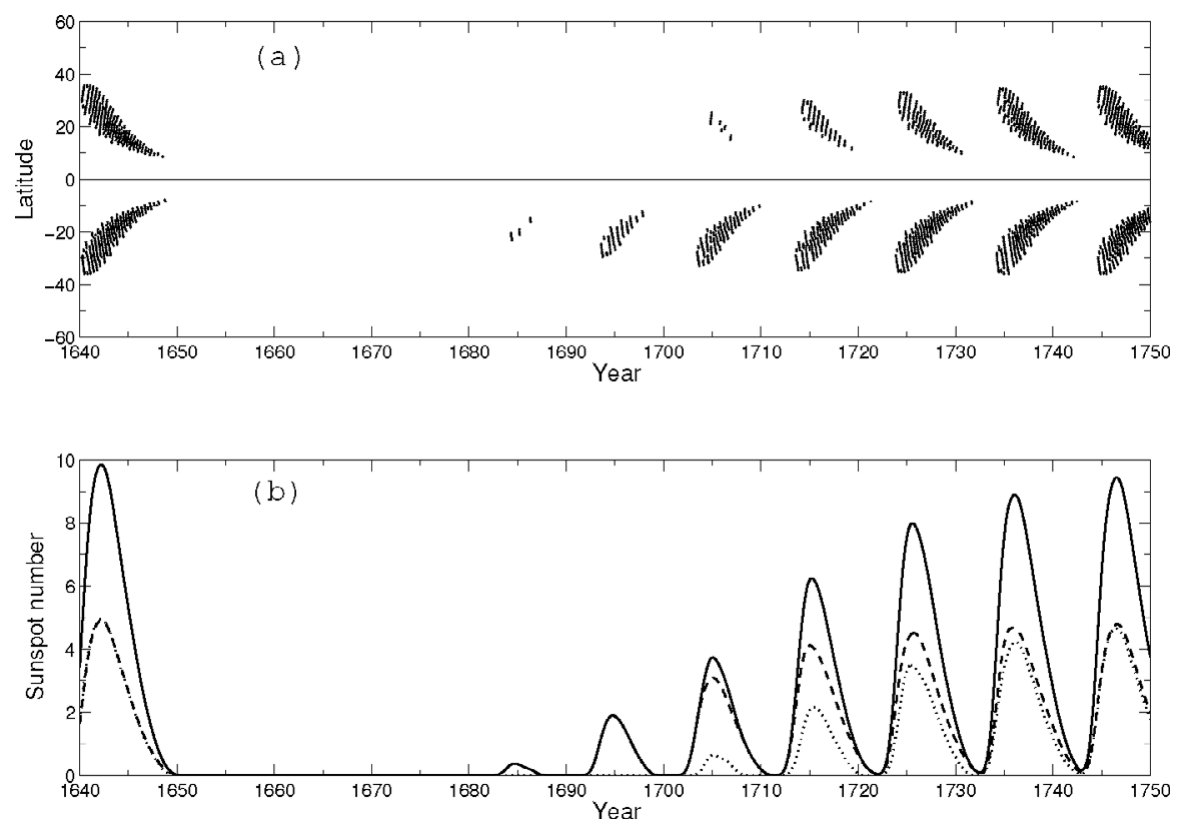

Figure 1. Results covering the Maunder minimum episode. (a) The butterfly diagram. (b) The smoothed sunspot number. The dashed and dotted lines show the sunspot numbers in southern and northern hemispheres, whereas the solid line is the total sunspot number. From Choudhuri \& Karak (2009).

we decrease the toroidal field by multiplying it everywhere by 0.8 to stop the eruption for some time. This essentially reduces the strong overlap between two cycles in our model (see figure 13 of Chatterjee et al. 2004). After making these changes, we run the model for several cycles without any further change. In the second procedure for reproducing the Maunder minimum, we decrease the amplitude of MC $v_{0}$ abruptly to a very low value. After keeping it at low value for few years, we again increase it to the usual value but at different rates in two hemispheres. In the northern hemisphere, it is increased at slightly lower rate than the southern hemisphere. Note that in this case we have varied only $v_{0}$ and no other parameters of the model. We have repeated this calculation in the low diffusivity model of Dikpati \& Charbonneau (1999) too. Last, we have included the effect of the fluctuations of polar field along with the fluctuations of MC. We have run the model for different values of $\gamma_{\mathrm{s}}$ from 0 to 1 at each values of $v_{0}$ from a very low value to the average value. Then we find out the critical values of $v_{0}$ and the corresponding $\gamma$ factor for which we get a Maunder-like minimum.

\section{Results}

First, we discuss the results from the polar field reduction procedure. It is shown in Fig. 1 (see the caption also). In order to facilitate the comparison with the observation data, we have marked the beginning of Fig. 1 to be the year 1640. From this figure we see that the sudden initiation but gradual recovery of Maunder minimum and the north-south asymmetry of sunspot numbers in the last phase have been nicely reproduced. We also find the cyclic oscillation of the poloidal field in the solar wind (shown in figure 2 of Choudhuri \& Karak 2009). This oscillation explains the cyclic behavior found 


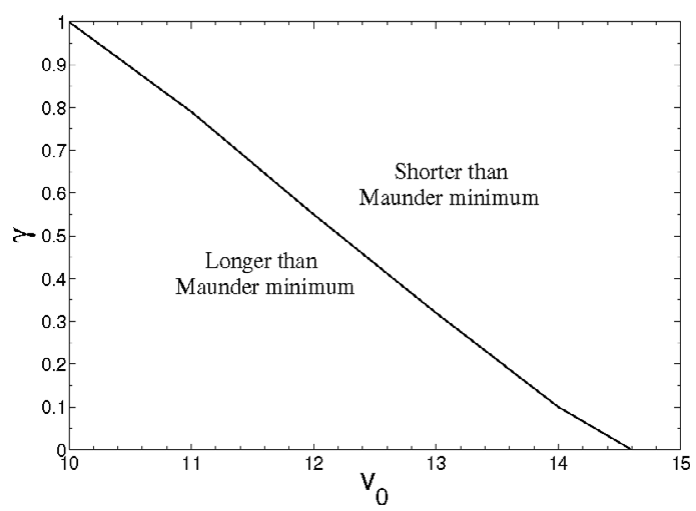

Figure 2. Parameter space of amplitude of MC $\left(v_{0}\right)$ and polar field reduction factor $(\gamma)$. The line shows the values of these parameters which are giving Maunder-like grand minima.

in cosmogenic isotopes data. In this calculation, we have taken $\alpha=21 \mathrm{~m} \mathrm{~s}^{-1}$ and turbulent diffusivity $\eta_{p}$ of the poloidal field within the convection zone $=3.2 \times 10^{12}$ $\mathrm{cm}^{2} \mathrm{~s}^{-1}$. This combination of $\alpha$ and $\eta_{p}$ gives the correct growth rate to produce Maunder minimum. In Choudhuri \& Karak (2009), we list some other combinations which also reproduce Maunder-like grand minima.

In the second procedure of reducing $v_{0}$ alone, we again get results very similar to what are shown in Fig. 1, if $v_{0}$ is abruptly made $10 \mathrm{~m} \mathrm{~s}^{-1}$ and then allowed to gain back its strength (see figures in Karak 2010). This procedure also reproduces all the important features of the Maunder minimum remarkably well. We underscore that in the advectiondominated model (e.g. Dikpati \& Charbonneau 1999) we do not get this result. This is because the decrease of MC in the advection-dominated model produces more toroidal field and makes the cycle stronger, as analyzed by Yeates et al. (2008).

We conclude that it is possible to reproduce the Maunder minimum by decreasing either polar field or $v_{0}$ to very low values. However, if we allow both the polar field and $v_{0}$ to decrease simultaneously, then it seems possible to reproduce Maunder-like grand minima without making either the polar field or the MC so low. We have found that for each $\gamma$ there is a particular value of $v_{0}$ which can produce a Maunder-like minimum. The line in Fig. 2 shows the combinations of parameters giving a Maunder minimum of appropriate duration. Values of $\gamma$ and $v_{0}$ lying in the lower left of Fig. 2 give grand minima longer than the Maunder minimum, whereas values lying in the upper right give shorter minima. We should mention that Fig. 1 is reproduced just by taking $\gamma=0.2$ (actually $\gamma_{N}=0.0$ and $\gamma_{S}=0.4$ is used) keeping $v_{0}$ unchanged. However, in this calculation, we have to reduce $v_{0}$ to around $13.5 \mathrm{~m} \mathrm{~s}^{-1}$ along with the polar field reduction to $0.2 \mathrm{in}$ both hemispheres. This is because in earlier calculations we had reduced the toroidal field slightly along with the change of polar field. Here we have not done this reduction of the toroidal field. Additionally, here the values of $\alpha$ and $\eta_{p}$ are slightly different giving different dynamo growth rate.

\section{Conclusion}

We have shown that most of the important features of the Maunder minimum can be reproduced quite well by assuming a simple ansatz that the polar field or the amplitude of MC or both decreased significantly at the beginning of Maunder minimum. Because of our lack of knowledge about the physical conditions at the beginning of the Maunder 
minimum, we cannot say how exactly the Sun was driven to the Maunder minimum. However, we should mention that there are several independent studies (Wang \& Sheeley 2003; Miyahara et al. 2004; Passos \& Lopes 2011) suggesting that the amplitude of MC was weaker during the Maunder minimum. If this happens to be correct, then this study along with several other studies (Chatterjee et al. 2004; Chatterjee \& Choudhuri 2006; Jiang et al. 2007; Yeates et al. 2008; Goel \& Choudhuri 2009; Karak \& Choudhuri 2011) indicate that the solar dynamo actually is diffusion-dominated and not advectiondominated.

\section{References}

Chatterjee, P. \& Choudhuri, A. R. 2006, Solar Phys., 239, 29

Chatterjee, P., Nandy, D., \& Choudhuri, A. R. 2004, Astron. Astrophys, 427, 1019

Choudhuri, A. R. 1992, Astron. Astrophys, 253, 277

Choudhuri, A. R., Chatterjee, P., \& Jiang, J. 2007, Phys. Rev. Lett., 98, 131103

Choudhuri, A. R. \& Karak, B. B. 2009, RAA, 9, 953

Choudhuri, A. R., Schüssler, M., \& Dikpati, M. 1995, Astron. Astrophys, 303, L29

Dikpati, M. \& Charbonneau, P. 1999, Astrophys. J., 518, 508

Durney, B. R. 1995, Solar Phys., 160, 213

Goel, A. \& Choudhuri, A. R. 2009, RAA, 9, 115

Hathaway, D. H. 1996, Astrophys. J., 460, 1027.

Jiang, J., Chatterjee, P., \& Choudhuri, A. R. 2007, Mon. Not. Roy. Astron. Soc., 381, 1527

Hoyt, D. V. \& Schatten, K. H. 1996, Solar Phys., 165, 181

Karak, B. B. 2010, Astrophys. J., 724, 1021

Karak, B. B. \& Choudhuri, A. R. 2011, Mon. Not. Roy. Astron. Soc., 410, 1503

Passos, D. \& Lopes, I. P. 2011, JASTP, 73, 191

Ribes, J. C. \& Nesme-Ribes, E. 1993, Astron. Astrophys, 276, 549

Wang, Y. -M. \& Sheeley, N. R. Jr. 2003, Astrophys. J., 591, 1248

Yeates, A. R., Nandy, D., \& Mackay, D. H. 2008, Astrophys. J., 673, 544 